\title{
Cell block technique as a complementary method in the clinical diagnosis of cyst-like lesions of the jaw
}

\author{
Elena Riet Correa RIVERO'1, Liliane Janete GRANDO¹, Fabíola MENEGAT², Jonathas Daniel Paggi CLAUS³, \\ Felipe Marcelo XAVIER ${ }^{4}$
}

\begin{abstract}
1- DDS, PhD, Assistant Professor, Department of Pathology, Health Science Center, Federal University of Santa Catarina, Florianópolis, SC, Brazil. 2- DDS, Health Science Center, Federal University of Santa Catarina, Florianópolis, SC, Brazil.

3- DDS, Resident in Surgery and Oral and Maxillofacial Traumatology, University Hospital, Federal University of Santa Catarina, Florianópolis, SC, Brazil. 4- MD, Nereu Ramos Hospital, Florianópolis, SC, Brazil.
\end{abstract}

Corresponding address: Elena Riet Correa Rivero - Departamento de Patologia - Centro de Ciências da Saúde - Universidade Federal de Santa Catarina - Florianópolis-SC, Brasil - 88040-370 - Phone: +55-48-3721-5068 - e-mail: riet@ccs.ufsc.br

Received: July 1, 2009 - Modification: March 2, 2010 - Accepted: April 29, 2010

\section{ABSTRACT}

\begin{abstract}
bjective: To evaluate the applicability of the cell block technique as a complementary method for presumptive diagnosis in the analysis of cyst-like aspirates from jaw lesions prior to histopathological diagnosis. Material and Methods: The sample was made up of 17 cyst-like jaw lesions. After puncture, the aspirates were centrifuged, fixed in formalin, embedded in paraffin and processed. All lesions were biopsied and submitted to histopathological examination. Results: In 9 cases, the cytopathological analysis using the cell block method showed a predominant presence of erythrocytes, inflammatory cells and few epithelial cells. In the other 8 cases, the cell block technique demonstrated the presence of parakeratin, whose histopathological analysis confirmed the occurrence of keratocystic odontogenic tumors (KOTs). Conclusions: According to the studied cases, the cell block method was proven to be a simple, fast and low-cost technique to effectively differentiate KOTs from other lesions with similar clinical and radiographic features. The cell block technique comprises cellular preparations useful to determine a clinical diagnosis and help to develop a therapeutic plan for those lesions.
\end{abstract}

Key words: Cytology. Biopsy. Jaw cysts. Odontogenic cysts. Odontogenic tumors.

\section{INTRODUCTION}

Odontogenic cysts of the jaw are lesions lined by epithelium of odontogenic origin that contain liquid or semi-solid material in their interior. These lesions have an autonomous growth owing to a difference in pressure. Radiologically they are characterized by a homogeneous radiolucent image, usually round or ovoid and well delineated by sclerotic margins ${ }^{18}$.

Despite having peculiar clinical and radiographic characteristics, the differential diagnosis of odontogenic cysts and tumors can be challenging for dental physicians, radiologists and surgeons, and it is of great importance for a definitive diagnosis establishment and treatment planning.

In the latest World Health Organization $(\mathrm{WHO})^{2}$ classification, some lesions previously considered as cystic are now classified as cystic tumors, once the growth mechanism does not occur due to a difference in pressure, but rather for other reasons, such as epithelium activity proliferation. This is the case of the keratocystic odontogenic tumor (KOT), formerly known as odontogenic keratocyst. Epithelial lining proliferation of KOT has a higher rate than non-odontogenic and radicular cysts, and is similar to the one found in ameloblastoma and the dental lamina7. Immunohistochemical staining for Ki-67 proliferation antigen shows numerous cells in cycle, not only in the basal, but also in the suprabasal epithelial KOT regions?.

This change in denomination made by WHO reflects the neoplastic nature of the lesion, due to its aggressive potential, infiltrating behavior through the medullar cavity of jaw, high recurrence rates 
and association with the nevoid basal cell carcinoma syndrome ${ }^{3,7}$.

KOT tends to grow occupying the anteroposterior medullar spaces, thus causing extensive lesions with little or no bone expansion. It is more commonly located in the posterior region of the mandible, presents scalloped radiographic margins, can displace teeth and usually does not reabsorb roots ${ }^{17}$. It is worth emphasizing that, radiographically, KOT may mimic a number of other odontogenic jaw cysts, or even appear as a lesion with a multilocular aspect. It is important, then, to include this tumor in the differential diagnosis of jaw radiolucent lesions, either unilocular or multilocular ${ }^{1,15}$.

A suitable semiotechnique method to help determining the clinical and differential diagnosis between jaw cystic and tumoral lesions is aspiration puncture. The aspiration of a cystic lesion can provide additional information about its content (if liquid or serous, or if absent) and aid in the presumptive clinical diagnosis at the moment of its application ${ }^{5}$. Cell block is a histological technique largely used in medical pathology for pleural fluid, peritoneal fluid, bronchial washings, fine-needle aspirations, and other cytological specimens ${ }^{9}$. Cell blocks prepared from residual tissue fluids and fineneedle aspirations can be useful adjuncts to smears for establishing a more definitive diagnosis ${ }^{13}$. The great advantage of cell block elaboration is the decrease in cellular dispersion - characteristic of tissue fluids - through centrifugation and its embedment in paraffin, which allows a better analysis of the collected liquid content, as well as the obtention of fine sections for analysis ${ }^{8}$.

Cell block is still rarely referred to in dental literature. Nevertheless, due to the fact that jaw cystic lesions are similar to cysts located elsewhere in the human body and also have dispersed cells in a liquid content, it offers a timely and appropriate application. The aim of this research was to evaluate the viability of cell block technique as a complementary method for the presumptive diagnosis starting from the collected material from lesions with clinical diagnosis of jaw cysts.
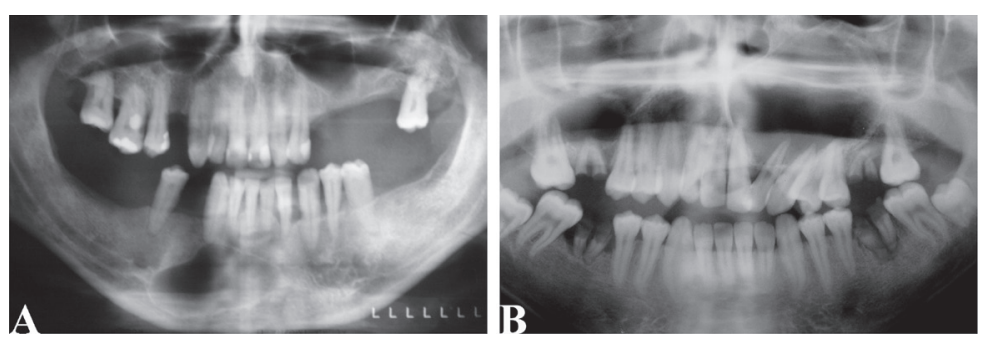

Figure 1- Panoramic radiographs: A, classical cyst aspect as a unilocular radiolucency lesion with well-defined sclerotic margins in a mandible residual cyst; B, maxillary keratocystic odontogenic tumor with unusual bone expansion and tooth displacement

\section{MATERIAL AND METHODS}

The project that originated this study was approved by the Research Ethics Committee of the Federal University of Santa Catarina (UFSC), registration number 075/2006.

The sample was made up of 17 patients with cyst-like jaw lesions that had indication for fine needle aspiration biopsy, being assisted at the Stomatology, Oral and Maxillofacial Traumatology Clinics of the University Hospital of Federal University of Santa Catarina, Brazil. All participants were volunteers who were informed about the study purposes and were asked to sign an informed consent form.

Submucous lesions were submitted to asepsis, local anesthesia, and puncture by an $18 \mathrm{~g}$ (gauge) needle connected to a $10 \mathrm{~mL}$ syringe. The syringe containing the material collected was immediately stored in a container with ice and put aside for laboratory procedures.

In the laboratory, the syringe material was transferred to a test tube and centrifuged at 2,000 rpm for $20 \mathrm{~min}$. The pellet obtained thereby in a $10 \%$ formaldehyde solution for $24 \mathrm{~h}$. After that, the material was sequentially processed as follows: dehydration, clearing, impregnation by and embedment in paraffin. Five-micrometerthick sections were obtained and stained with hematoxylin and eosin (H\&E).

Specimens were analyzed by light microscopy (Axiostar Plus; Carl Zeiss, Oberkochen, Germany) to characterize the type of material collected in relation to the presence of epithelial cells, inflammatory cells, keratin, erythrocytes, or any other components that could be present.

All patients who participated in this study were submitted to appropriate radiographic exams and incisional or excisional biopsy, in accordance with clinical indication.

The material from biopsies was processed and stained with H\&E. Results from the histological examination of the cell block were compared with the histopathological data of the biopsy, as well with the clinical and radiographic data of the lesions. The was transferred onto absorbent paper and fixed 
analysis of the clinical, radiographic and cell block data, combined with histopathological examination of the biopsied tissue allowed determining the lesion diagnosis. All patients were treated properly and were followed by clinical/radiographic postoperative control.

\section{RESULTS}

Cell blocks of 17 cyst-like jaw lesions were performed. Based on clinical and radiographic images all lesions were diagnosed as inflammatory cyst or KOT. The radiograph findings showed unilocular radiolucency lesions with well-defined sclerotic margins (Figures $1 \mathrm{~A}$ and $\mathrm{B}$ ). In 9 cases $(53 \%)$, cytological analysis revealed a large number of erythrocytes, several inflammatory cells, and a small number of epithelial cells, with cholesterol clefts in some cases (Figures 2A and B). Histopathological analysis associated with clinical and radiographic characteristics rendered the diagnosis of inflammatory odontogenic cysts: 3 residual cysts and 6 radicular cysts (Figures 2C and D). In 8 cases (47\%), diagnosed as KOTs by
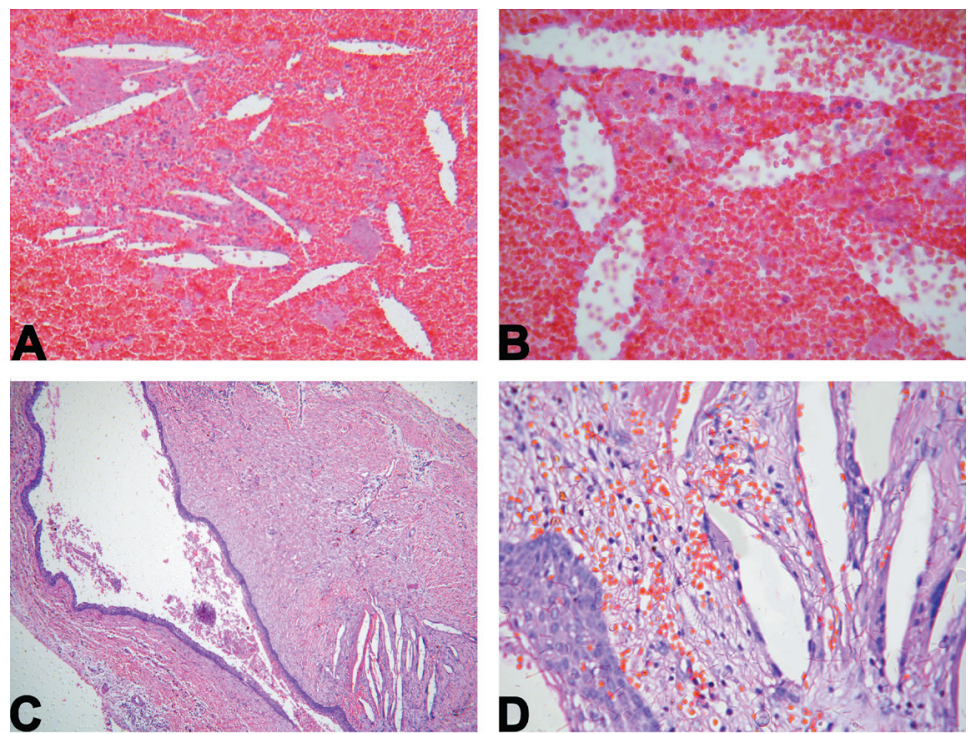

Figure 2- A, cytological analysis (cell block) of an inflammatory cyst [hematoxylin-eosin (H\&E)100x]; B, high magnification showing cholesterol clefts and a large number of erythrocytes (H\&E 400x); C, histopathological analysis of the same lesion showing a cystic capsule with epithelial lining (H\&E 100x); D, high magnification showing cholesterol clefts inside the capsule and the stratified epithelial lining (H\&E 400x)
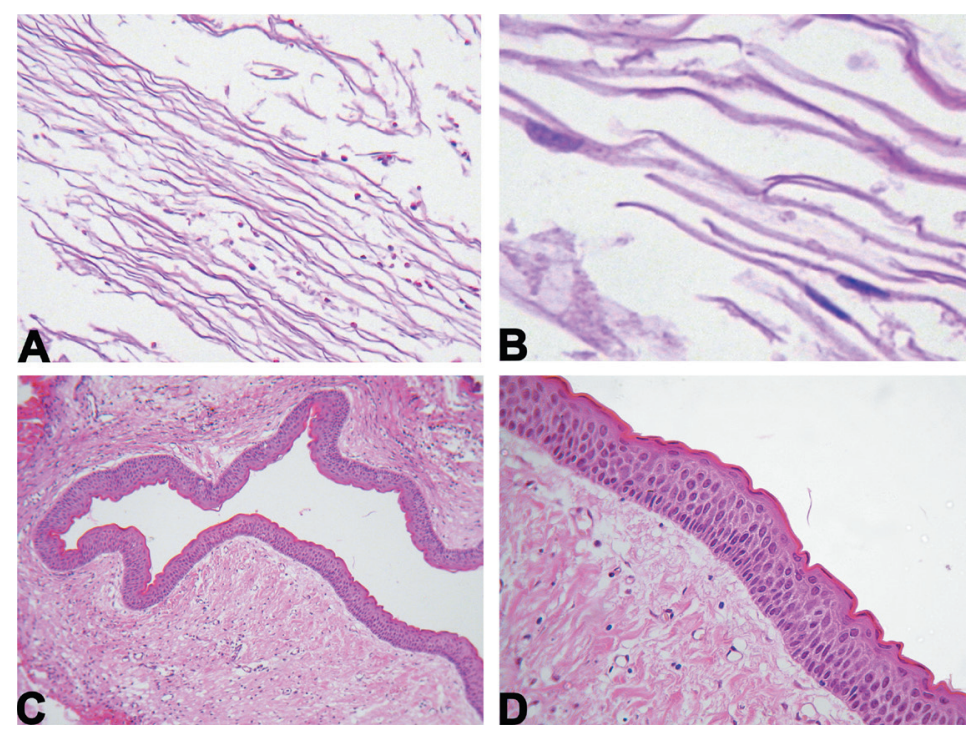

Figure 3-A, cytological analysis (cell block) of keratocystic odontogenic tumor showing keratin [hematoxylin-eosin (H\&E)100x] and, B, parakeratin (high magnification) (H\&E 1,000x); C, histopathological analysis of the same lesion with a conjunctive tissue capsule lined by thin and uniform epithelial tissue (H\&E 100x); D, high magnification showing the stratified epithelial lining with a palisade basal layer and corrugated parakeratin on the surface (H\&E 400x) 
the histopathological analysis, the cell block method showed a predominant occurrence of keratin and the presence of parakeratin in some areas (Figures $3 A, B, C$ and $D$ ).

\section{DISCUSSION}

Differential diagnosis of cystic lesions can be rather difficult. Therefore, establishing a correlation between clinical, radiographic, cytological, and histopathological characteristics is essential for diagnosis and subsequently for the best treatment planning for each specific case.

Jaw cysts and benign osseous tumors have a similar behavior in relation to evolution period (slow), expansion of the bone, eventual dental displacement, and even dental resorption ${ }^{17}$. In general, their radiographic aspects can be confusing, and it is not always possible to make an accurate clinical diagnosis among them ${ }^{15}$.

Although the fine needle aspiration biopsy is a semiotechnique method frequently used to analyze cyst-like lesion content, in most of the cases the collected material is discarded. Cell block is a histological technique that allows the microscopic analysis of this material. The advantage of using the cell block technique is the decrease in cellular dispersion, in an attempt to obtain maximum cellular concentration, which facilitates microscopic analysis. Another advantage is the fact that the material can be stored for future use, if necessary, as in immunohistochemical essays ${ }^{10,12}$.

According to the studied cases, the cell block method proved to be very effective in distinguishing KOTs from other lesions with similar clinical and radiographic aspects, through parakeratin identification. The inflammation could affect the walls KOT causing epithelium metaplasia and loss of their peculiar characteristics7. Especially in cases of incisional biopsy, the small selected area could indicate epithelial alterations and cause difficulties in the histological diagnosis. In case of large lesions, the cell block method also has the advantage of detecting the presence of parakeratin, as it was observed in one of our cases, where the histopathological analysis showed a severely inflamed area.

Some authors have report that keratocysts behave as benign neoplasm and should be treated as such ${ }^{12}$. However, KOTs tend to grow, occupying the medullar cavity of bone without causing a significant expansion and frequently reaching a considerable size, particularly in the mandible ramus and angle. Due to the fact that there is not enough information about the lesion development period before its diagnosis, the recent classification of the odontogenic keratocyst as a KOT by $\mathrm{WHO}^{2}$ shows the importance of establishing a correct

\section{diagnosis.}

Kramer and Toller $^{11}$ (1973) mentioned that the presence of keratin in the cystic content is a strong evidence that the lesion is a KOT. Currently, it is known that parakeratin is the main substance found in this lesion, and that orthokeratin focuses can also occur ${ }^{7}$. Cysts with a great amount of orthokeratin are called orthokeratinized odontogenic cysts and have low aggressive potential and minimum recurrence rate ${ }^{6}$. This kind of cyst is a rare lesion and, although the result of cell bock may be similar, they do not present parakeratin, as in all KOT cases of this study.

A good exemplification of how cell block preparations can be very useful in the differential diagnosis of cyst-like jaw lesions occurred in one of the reported cases of KOT (Figure 1B). This lesion had an unusual behavior for a KOT, causing bone expansion, dental displacement, and displacement of the maxillary sinus floor, and it was clinically diagnosed as an infected radicular cyst. The clinical aspiration procedure showed a whitish and pasty material, compatible with KOT, and this clinical diagnosis was confirmed by the presence of parakeratin in the cell block and later by its typical histopathological characteristics.

It is also important to mention that some benign tumors may present a cystic degeneration, such as ameloblastoma, ameloblastic fibroma and odontogenic adenomatoid tumor ${ }^{4,14,16}$, and consequently, liquid content in their interior. Cell block method can be used for differential diagnosis from these lesions.

\section{CONCLUSIONS}

The cell block method is a simple, fast and lowcost technique that can be used as an adjunctive technique in the presumptive diagnosis of jaw cystic lesions. Embedded cell preparations by the cell block technique from fine needle aspiration biopsy are useful for establishing a differential diagnosis between KOT and jaw lesions with cystic aspects, aiding the therapeutic planning of these lesions.

\section{REFERENCES}

1- Ali M, Baughman RA. Maxillary odontogenic keratocyst: a common and serious clinical misdiagnosis. J Am Dent Assoc. 2003;134(7):877-83.

2- Barnes L, Eveson JW, Reichart PA, Sidransky D, eds. WHO classification of tumors. Pathology and genetics of head and neck tumours. Lyon: IARC Press; 2005.

3- Bell RB, Dierks EJ. Treatment options for the recurrent odontogenic keratocyst. Oral Maxillofac Surg Clin North Am. $2003 ; 15(3): 429-46$.

4- Choudhury M, Dhar S, Bajaj P. Primary diagnosis of ameloblastoma by fine-needle aspiration: a report of two cases. Diagn Cytopathol. 2000;23(6):414-6.

5- Coleman GC, Nelson JF. Principles of oral diagnosis. Saint Louis: Mosby-Year Book; 1993. 
6- Dong Q, Pan S, Sun LS, Li TJ. Orthokeratinized odontogenic cyst: a clinicopathologic study of 61 cases. Arch Pathol Lab Med. 2010;134(2):271-5.

7- Jordan RC. Histology and ultrastructural features of the odontogenic keratocyst. Oral Maxillofac Surg Clin North Am. 2003;15(3):325-33.

8- Karnauchow PN, Bonin RE. "Cell-block" technique for fine needle aspiration biopsy. J Clin Pathol. 1982;35(6):688.

9- Keyhani-Rofagha S, Vesey-Shecket M. Diagnostic value, feasibility, and validity of preparing cell blocks from fluid-based gynecologic cytology specimens. Cancer. 2002;96(4):204-9.

10- Khurana KK, Ramzy I, Truong LD. p53 immunolocalization in cell block preparations of squamous lesions of the neck: an adjunct to fine-needle aspiration diagnosis of malignancy. Arch Pathol Lab Med. 1999;123(5):421-5.

11- Kramer IR, Toller PA. The use of exfoliative cytology and protein estimations in preoperative diagnosis of odontogenic keratocysts. Int J Oral Surg. 1973;2(4):143-51.
12- Mayall F, Chang B, Darlington A. A review of 50 consecutive cytology cell block preparations in a large general hospital. J Clin Pathol. 1997;50(12):985-90.

13- Nathan NA, Narayan E, Smith MM, Horn MJ. Cell block cytology. Improved preparation and its efficacy in diagnostic cytology. Am J Clin Pathol. 2000;114(4):599-606.

14- Nigam S, Gupta SK, Chaturvedi KU. Adenomatoid odontogenic tumor-a rare cause of jaw swelling. Braz Dent J. 2005;16(3):2513.

15- Raitz R, Assunção Júnior JN, Correa L, Fenyo-Pereira M. Parameters in panoramic radiography for differentiation of radiolucent lesions. J Appl Oral Sci. 2009;17(5):381-7.

16- Reichard PA, Philipsen, HP. Odontogenic tumours and allied lesions. London: Quintessence Publishing Company; 2004.

17- Shear M. Odontogenic keratocysts: clinical features. Oral Maxillofacial Surg Clin N Am. 2003;15(3):335-45.

18- Varinauskas V, Gervickas A, Kavoliuniene O. Analysis of odontogenic cysts of the jaws. Medicina (Kaunas). 2006;42(3):2017. 